\title{
Prospects for the detection of heavy charginos and neutralinos with the ATLAS detector at the LHC
}

\author{
Giacomo Polesello \\ INFN, Sezione di Pavia Via Bassi 6, I-27100 Pavia, Italy
}

\begin{abstract}
The high cross-section for squark and gluino production at the LHC allows the search for rare cascade decays of the squarks, thus giving access to a large spectrum of SUSY sparticles. In the framework of minimal SUGRA we investigate the possibility of detecting the heavier gaugino states $\tilde{\chi}_{4}^{0}$ and $\tilde{\chi}_{2}^{ \pm}$through their decays to sleptons. The characteristic final state signature of two leptons with opposite sign and same flavour allows the signal to be discriminated from the dominant SUSY background, yielding a significant reach in the mSUGRA parameter space.

The shape of the lepton-lepton invariant mass distribution for the observed signal can be used to extract a measurement of the masses of the heavier gauginos.
\end{abstract}




\section{Introduction}

If supersymmetry is discovered, the main task of the experiments will be the unravelling of the pattern of supersymmetry breaking through a measurement of the model parameters at the weak scale.

The study of the production and decay of the superpartners of the gauge bosons is a privileged path toward the achievement of this program. Consider the neutral gauginohiggsino sector for example. In the Minimal Supersymmetric Standard Model (MSSM), assuming a $C P$-conserving scenario, the SUSY partners of the neutral gauge and higgs bosons:

$$
\left(\tilde{B}, \tilde{W}^{3}, \tilde{H}_{1}^{0}, \tilde{H}_{2}^{0}\right)
$$

mix to form the mass eigenstates $\chi_{i}^{0}(i=1,2,3,4)$ through the matrix [1]:

$$
\mathcal{M}=\left(\begin{array}{cccc}
M_{1} & 0 & -m_{Z} \cos \beta s_{W} & m_{Z} \sin \beta s_{W} \\
0 & M_{2} & m_{Z} \cos \beta c_{W} & -m_{Z} \sin \beta c_{W} \\
-m_{Z} \cos \beta s_{W} & m_{Z} \cos \beta c_{W} & 0 & -\mu \\
m_{Z} \sin \beta s_{W} & -m_{Z} \sin \beta c_{W} & -\mu & 0
\end{array}\right)
$$

Where $M_{1}$ and $M_{2}$ are respectively the $\mathrm{U}(1)$ and $\mathrm{SU}(2)$ gaugino masses, $\mu$ is the higgsino mass parameter, $\tan \beta=v_{2} / v_{1}$ is the ratio of the vacuum expectation values of the two higgs doublets of the model, $s_{W}$ and $c_{W}$ are respectively the sine and cosine of the electroweak mixing angle $\theta_{W}$, and $m_{Z}$ is the mass of the $Z$ boson. The fundamental parameters entering the mixing matrix will be thus constrained through the measurement of the gaugino masses and branching ratios.

Following the previous detailed studies of SUSY phenomenology at the LHC, we concentrate here on the constrained version of the MSSM where supersymmetry breaking is mediated by the gravitational interaction (mSUGRA). In this model all the masses and couplings of the sparticles are determined by four parameters: $m_{0}$ and $m_{1 / 2}$, respectively the universal scalar mass and the universal gaugino mass at GUT scale, a universal trilinear term $A, \tan \beta$ and the sign of $\mu$. We use the specific implementation of the model available through the ISASUSY program [4]. In mSUGRA, for the range of parameters relevant for this analysis, $|\mu|>M_{2}$. After diagonalization of the gaugino mixing matrices the lighter charginos/neutralinos are essentially gauginos and precisely $\tilde{\chi}_{1}^{0} \sim \tilde{B}, \tilde{\chi}_{2}^{0} \sim \tilde{W}^{3}, \tilde{\chi}_{1}^{ \pm} \sim \tilde{W}^{ \pm}$, whereas the heavier ones $\tilde{\chi}_{3}^{0}, \tilde{\chi}_{4}^{0}$, and $\tilde{\chi}_{2}^{ \pm}$are almost pure higgsinos. Therefore the measurement of the masses of the lighter gauginos mostly constrains the $M_{1}$ and $M_{2}$ parameters, and it only yields very loose constraints on the other elements of the mixing matrix. In order to resolve completely the matrix using only the lighter gauginos, additional detailed information on the gaugino production mechanisms is needed, for example from a future high energy $e^{+} e^{-}$collider [5].

At the LHC, most of the gauginos are produced in the decays of squarks and gluinos, and typically in mSUGRA,

$$
B R\left(\tilde{q}_{L} \rightarrow \tilde{\chi}_{2}^{0} q\right) \sim 30 \%, \quad B R\left(\tilde{q}_{L} \rightarrow \tilde{\chi}_{1}^{ \pm} q\right) \sim 60 \%
$$


so that the phenomenology and hence the experimental studies are dominated by the lighter gauginos $[2,3]$. The LHC will however provide a very high statistics sample, which for one year at high luminosity will correspond to a few $10^{5}$ SUSY events for squark/gluino masses of $\sim 1 \mathrm{TeV}$. It is therefore conceivable to try to study the rare decays of the squarks into the heavier gauginos.

After diagonalization of the mixing matrix in mSUGRA the $\tilde{\chi}_{3}^{0}$ is almost exclusively higgsino, with a very small gaugino component, but the $\tilde{\chi}_{4}^{0}$ has typically some gaugino admixture, yielding a significant coupling to the $\tilde{q}_{L}$ and a smaller, but significant coupling to the $\tilde{q}_{R}$. The same is true for the $\tilde{\chi}_{2}^{+}$. By taking as an example $\tan \beta=10$, $A=0 \mathrm{GeV}, \mu>0$, we show in Figures 1 and 2 the branching ratios of $\tilde{d}_{L}$ into respectively $\tilde{\chi}_{4}^{0}$ and $\tilde{\chi}_{2}^{ \pm}$in the $\left(m_{0}, m_{1 / 2}\right)$ plane, calculated with ISAJET [4].

As can be seen from the figures, the decays into heavy gauginos have a branching fraction of a only a few $\%$ over most of the parameter space. We therefore need to identify a clear signature which allows the separation of these decays from the overwhelming background from squarks decaying into the lighter gauginos.

For the $\tilde{\chi}_{2}^{0}$ measurement the best SUSY signature is the edge in lepton-lepton invariant mass from the decay chain [2]:

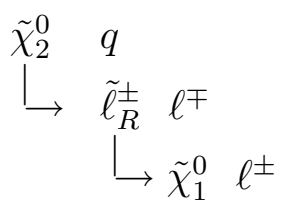

The clear kinematic structure gives a precise measurement of the kinematic end point of the lepton-lepton invariant mass. The dominant background from uncorrelated $\tilde{\chi}_{1}^{+}$ decays yields an equal number of Opposite-Sign Same-Flavour (OS-SF), and OppositeSign Opposite-Flavour (OS-OF) lepton pairs, whereas the signal only consists of OS-SF lepton pairs. A clean distribution of the lepton-lepton invariant mass for the signal can thus be obtained by subtracting the mass distribution for OS-OF pairs from the one for OS-SF pairs. A similar signature can be exploited for the decay of the heavier gauginos. The uncorrelated decays can still be subtracted using lepton flavour correlation, and the background from $\tilde{\chi}_{2}^{0}$ decays is eliminated by only considering lepton-lepton invariant masses above the $\tilde{\chi}_{2}^{0}$ kinematic edge.

Three decay chains for $\tilde{\chi}_{4}^{0}$ and one decay chain for $\tilde{\chi}_{2}^{ \pm}$do provide a signal with correlated lepton flavour:

$$
\begin{aligned}
& \tilde{\chi}_{4}^{0} \quad q \\
& \longrightarrow \tilde{\ell}_{R}^{ \pm} \ell^{\mp} \\
& \longrightarrow \tilde{\chi}_{1}^{0} \ell^{ \pm} \\
& \tilde{\chi}_{4}^{0} \quad q \\
& \longrightarrow \tilde{\ell}_{L}^{ \pm} \ell^{\mp} \\
& \longrightarrow \tilde{\chi}_{1}^{0} \quad \ell^{ \pm} \\
& \rightarrow \tilde{\chi}_{2}^{0} \quad \ell^{ \pm}
\end{aligned}
$$




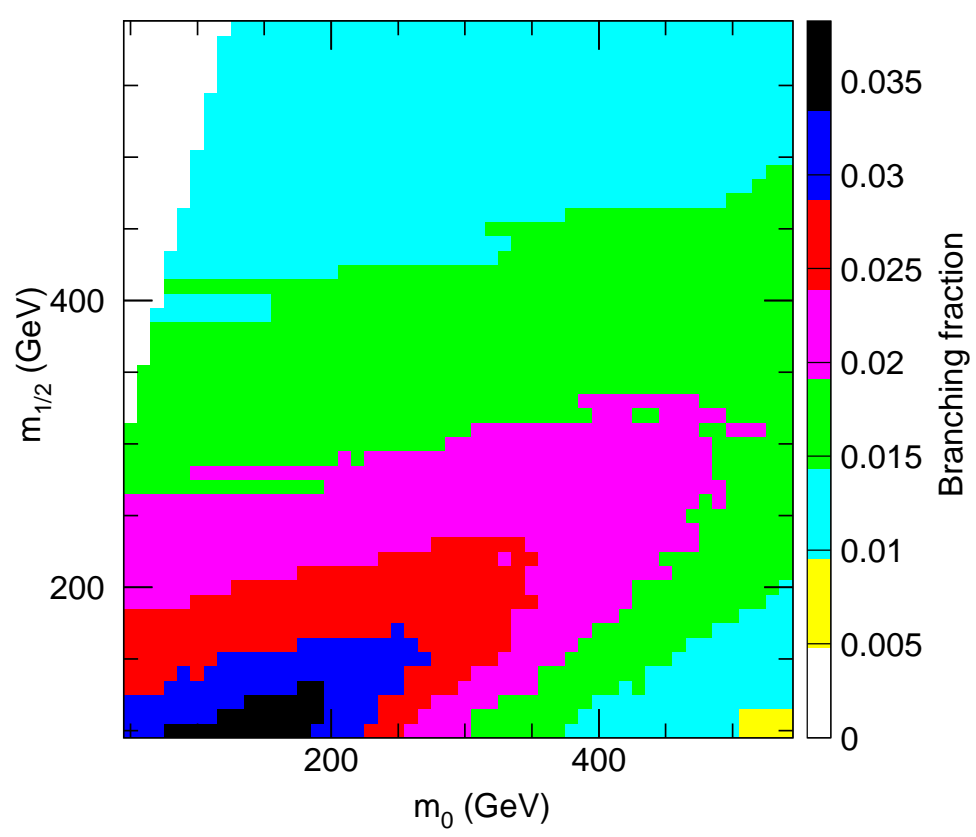

Figure 1. Branching fraction for the decay $\tilde{d}_{L} \rightarrow \tilde{\chi}_{4}^{0} d$ as a function of $m_{0}$ and $m_{1 / 2}$ for $\tan \beta=10, A=0$ and $\mu>0$.

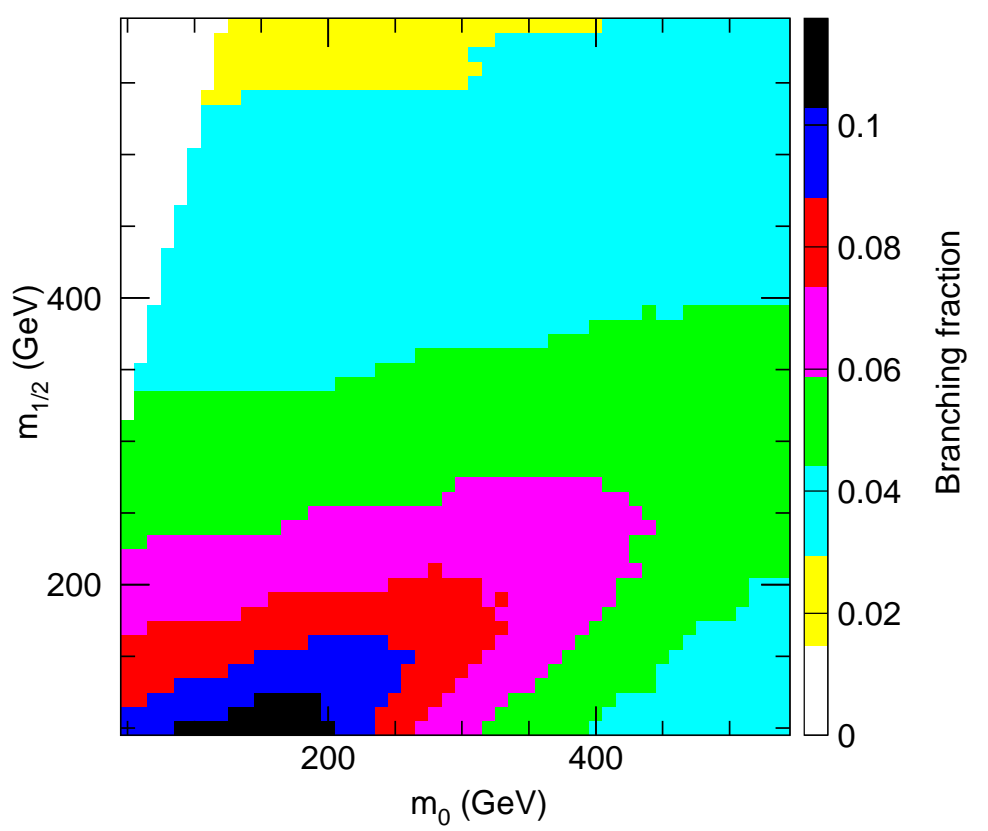

Figure 2. Branching fraction for the decay $\tilde{d}_{L} \rightarrow \tilde{\chi}_{2}^{-} u$ as a function of $m_{0}$ and $m_{1 / 2}$ for $\tan \beta=10, A=0$ and $\mu>0$. 
Table 1. Model points simulated for the present study. The last two columns give the total production cross-section in pb, and the number of generated events.

\begin{tabular}{lrrrrrrr}
\hline Point & $m_{0}$ & $m_{1 / 2}$ & $A$ & $\tan \beta$ & $\operatorname{sgn}(\mu)$ & $\sigma(\mathrm{pb})$ & $N_{e v} / 10^{6}$ \\
\hline A & 100. & 250. & -100. & 10. & + & 51.00 & 5 \\
B & 100. & 300. & 0. & 10. & + & 21.00 & 2 \\
C & 100. & 330. & 0. & 10. & + & 13.26 & 2 \\
D & 100. & 380. & 0. & 10. & + & 6.48 & 1 \\
E & 150. & 250. & 0. & 10. & + & 47.00 & 3 \\
F & 150. & 300. & 0. & 10. & + & 20.18 & 2 \\
G & 175. & 350. & 0. & 10. & + & 9.21 & 1 \\
H & 200. & 275. & 0. & 10. & + & 27.77 & 3 \\
I & 250. & 300. & 0. & 10. & + & 17.86 & 2 \\
L & 300. & 350. & 0. & 10. & + & 8.00 & 0.8 \\
\hline
\end{tabular}

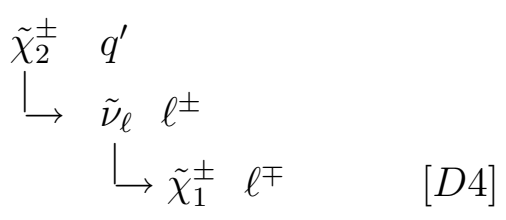

The coupling of the $\tilde{\chi}_{4}^{0}$ and of the $\tilde{\chi}_{2}^{ \pm}$to $\tilde{\ell}_{R}$ and $\tilde{\ell}_{L}$ is again determined by respectively the amount of bino and wino components in the gauginos. The branching ratios can be up to a few percent depending on the mSUGRA parameters.

The numbers of events produced in one year of high luminosity running at the LHC $\left(100 \mathrm{fb}^{-1}\right)$ containing the decay chains [D1]-[D4] were calculated using ISASUSY [4] for the branching ratios and PROSPINO [6] for the leading order production cross-section. The results for $A=0, \tan \beta=10$ and $\mu>0$, are shown as a function of $m_{0}$ and $m_{1 / 2}$ in Figures 3 and 4 . Two regions can be identified in the $\left(m_{0}, m_{1 / 2}\right)$ plane, one where $\tilde{q}_{L}$ only decays into $\tilde{\chi}_{4}^{0}$, another where both the decays into $\tilde{\chi}_{4}^{0}$ and $\tilde{\chi}_{2}^{ \pm}$are open, giving different shapes for the invariant mass spectra of the OS-SF lepton pairs.

\section{Event Simulation}

The SUSY events were generated with HERWIG 6.4 [7], and passed through ATLFAST [9], a parametrized simulation of the ATLAS detector.

The Standard Model backgrounds were generated with PYTHIA 6.1 [8]. The $Z+$ jets background can not be subtracted using the lepton subtraction procedure. Therefore for this channel the PYTHIA results were cross-checked using the ALPGEN generator [10] for the production of $Z+2$ jets. The two generators give statistically consistent estimates of the residual background after cuts. A complete analysis was performed for the ten points in mSUGRA parameter space shown in Table 1. Point A corresponds to the SPS1a Point [11], and Point B is a variation on Point 5 which is the reference model for most of the previous ATLAS studies.

The choice of the additional Points, shown as black dots in Figure 5 is meant to 


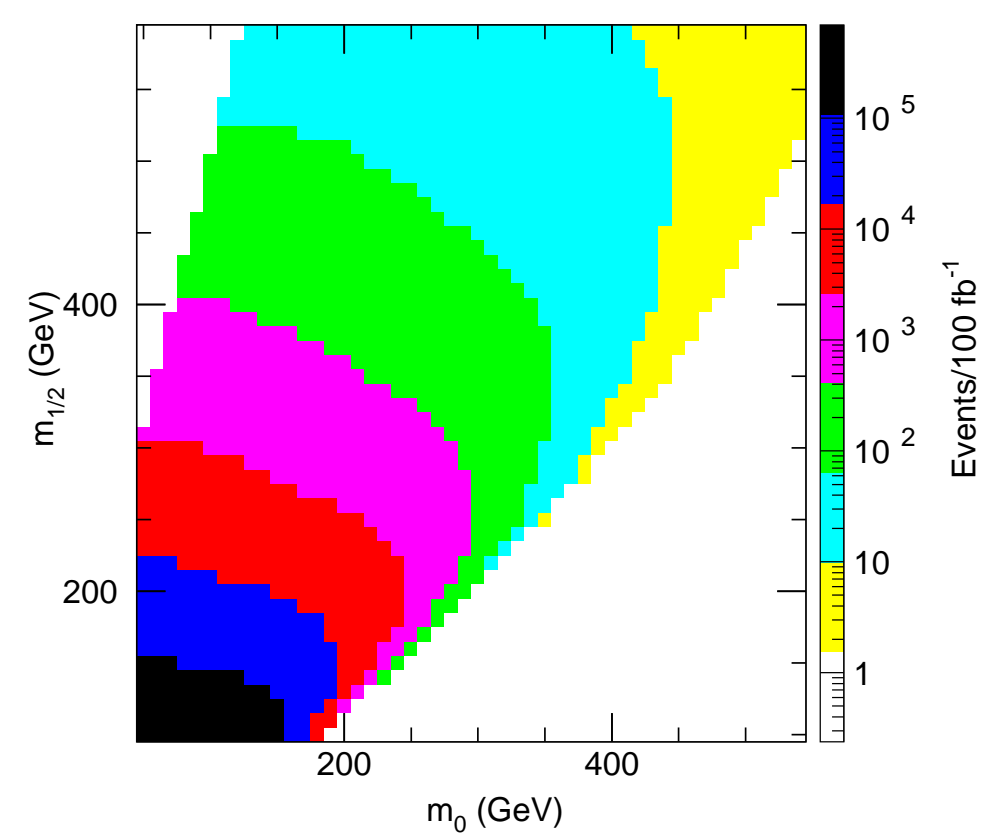

Figure 3. Expected number of events for one year at high luminosity $\left(100 \mathrm{fb}^{-1}\right)$ as a function $m_{0}$ and $m_{1 / 2}$ for the process: $p p \rightarrow \tilde{\chi}_{4}^{0}+X \rightarrow \ell^{+} \ell^{-}+X(\ell \equiv e$ or $\mu)$. Events where $\ell$ 's are produced in $\tau$ decays are not considered.

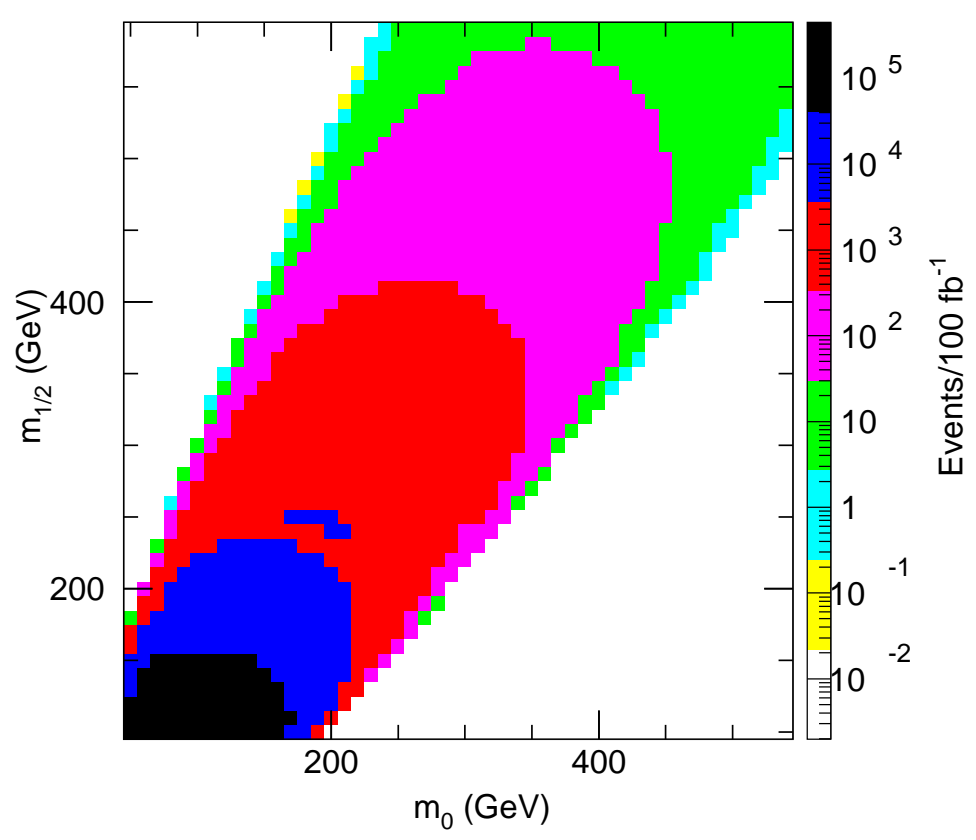

Figure 4. Expected number of events for one year at high luminosity $\left(100 \mathrm{fb}^{-1}\right)$ as a function $m_{0}$ and $m_{1 / 2}$ for the process: $p p \rightarrow \tilde{\chi}_{2}^{ \pm}+X \rightarrow \ell^{+} \ell^{-}+X(\ell \equiv e$ or $\mu)$. Events where $\ell$ 's are produced in $\tau$ decays are not considered. 


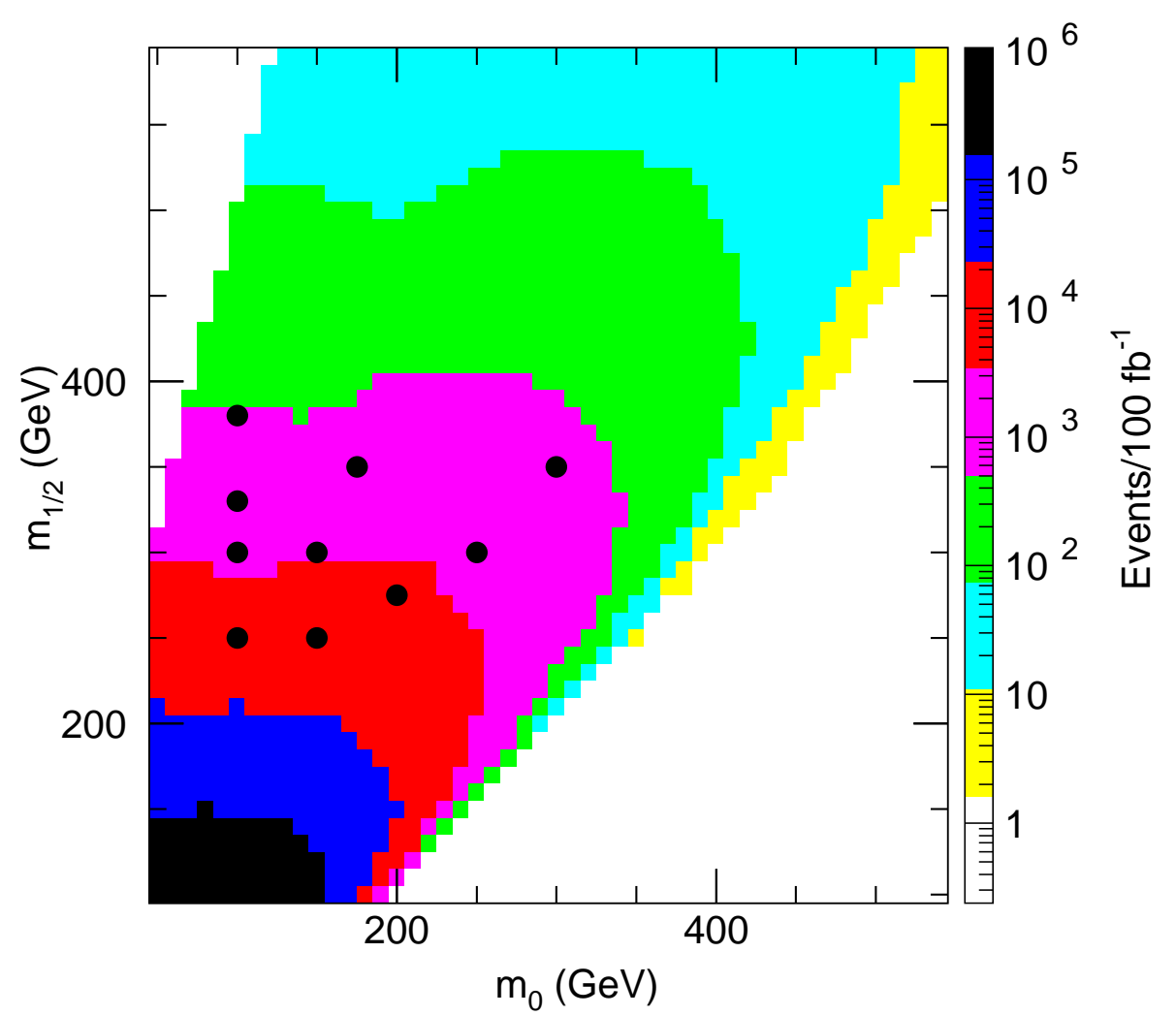

Figure 5. Expected number of events for one year at high luminosity $\left(100 \mathrm{fb}^{-1}\right)$ as a function $m_{0}$ and $m_{1 / 2}$ for the process: $p p \rightarrow \tilde{\chi}_{4}^{0}, \tilde{\chi}_{2}^{ \pm}+X \rightarrow \ell^{+} \ell^{-}+X(\ell \equiv e$ or $\mu)$. Events where $\ell$ 's are produced in $\tau$ decays are not considered. The black dots indicate the models studied in detail.

explore the reach of the ATLAS experiment for this signature, in particular following two directions in parameter space offering somewhat different phenomenologies:

- scan along $m_{0}=100 \mathrm{GeV}$ to study the region with dominant $\tilde{\chi}_{4}^{0}$,

- an approximate diagonal with significant $\tilde{\chi}_{2}^{ \pm}$signal.

The shape of the two-lepton invariant mass distribution for each of the [D1]-[D4] chains displays an edge, which, for each decay $p_{1} \rightarrow p_{2} \ell^{ \pm} \rightarrow p_{3} \ell^{ \pm} \ell^{\mp}$ is at the position $m_{l^{+} l^{-}}^{\max }$ given by the expression:

$$
m_{l^{+} l^{-}}^{\max }=m_{p_{1}} \sqrt{1-\frac{m_{p_{2}}^{2}}{m_{p_{1}}^{2}}} \sqrt{1-\frac{m_{p_{3}}^{2}}{m_{p_{2}}^{2}}}
$$

This is illustrated respectively in Figures 6 and 7, for Points A and E. The experimentally observed shape is the sum of the four shapes from the decays [D1] to [D4], shown in different colors in the figures, weighted by the relative production rates. Typically in mSUGRA, where $m_{\tilde{\chi}_{4}^{0}} \sim m_{\tilde{\chi}_{2}^{ \pm}}$, and $m_{\tilde{\chi}_{2}^{0}} \sim m_{\tilde{\chi}_{1}^{ \pm}}$, the edge from the decays [D3] and [D4] is at lower masses than the edge from [D1] and [D2]. The outermost edge, which 
gives the most easily measurable end point, is either from [D1] of [D2], depending on the position of the slepton masses in the $m_{\tilde{\chi}_{1}^{0}}-m_{\tilde{\chi}_{4}^{0}}$ interval. In fact, for Point $\mathrm{A}$ the outermost edge is from decay [D2], and for Point E it is from [D1].

A special case, illustrated in Figure 7 happens for the high $m_{0}$ region near the kinematic limit for the decay. In this case the $\tilde{\chi}_{2}^{ \pm}$decays are dominant, and the invariant mass shape can present a sharp edge from [D4] followed by a lower statistics tail from the $\tilde{\chi}_{4}^{0}$ decays.

\section{Data Analysis}

Given the low signal statistics a set of tight analysis requirements are applied in order to reduce the Standard Model background below the SUSY background from uncorrelated gaugino decays. We require:

- Exactly two isolated OS-SF leptons with transverse momenta $P_{T}(l 1)>20 \mathrm{GeV}$, $P_{T}(l 2)>10 \mathrm{GeV}$

- Total missing transverse momentum $P_{T}^{\text {miss }}>100 \mathrm{GeV}$

- At least four jets, $P_{T}(j 1)>150 \mathrm{GeV}, P_{T}(j 2)>100 \mathrm{GeV}, P_{T}(j 3, j 4)>20 \mathrm{GeV}$

- $M_{\text {eff }} \equiv P_{T}^{\text {miss }}+P_{T}(j 1)+P_{T}(j 2)+P_{T}(j 3)+P_{T}(j 4)>600 \mathrm{GeV}$

- $M_{T 2}>80 \mathrm{GeV}$

- $m_{\ell \ell}>100 \mathrm{GeV}$

The lepton isolation criterion consists in requiring a transverse energy deposition in the calorimeters smaller than $10 \mathrm{GeV}$ in a $(\eta, \phi)$ cone of radius 0.2 around the lepton direction, where $\eta$ is the pseudorapidity of the lepton and $\phi$ the angle in the plane transverse to the beam.

The variable $M_{T 2}$ is defined as:

$$
M_{T 2}^{2} \equiv \min _{\mathbf{p}_{1}+\mathbf{p}_{2}=\mathbf{p}_{T}}\left[\max \left\{m_{T}^{2}\left(\mathbf{p}_{T}^{l_{1}}, \mathbf{p}_{1}\right), m_{T}^{2}\left(\mathbf{p}_{T}^{l_{2}}, \mathbf{p}_{2}\right)\right\}\right]
$$

where the minimisation is performed over all the pairs of vectors in the transverse plane $\boldsymbol{p}_{1}, \boldsymbol{p}_{2}$ such that their vector sum is the missing transverse momentum $\boldsymbol{p}_{T}$, and

$$
\begin{aligned}
& m_{T}^{2}\left(\mathbf{p}_{T}, \mathbf{q}_{T}\right) \equiv 2\left(E_{T} Q_{T}-\mathbf{p}_{T} \cdot \mathbf{q}_{T}\right), \\
& E_{T}=\left|\mathbf{p}_{T}\right| \quad \text { and } \quad Q_{T}=\left|\mathbf{q}_{T}\right| .
\end{aligned}
$$

This variable is a special case of a variable first proposed in [12] and discussed in detail in [13]. The basic idea is that when an events contains two equal particles, both decaying to a neutrino and a visible particle, the maximum value that $M_{T 2}$ can assume is the mass of the parent particle. Therefore for $\bar{t} t$, the $M_{T 2}$ stops at $\sim 80 \mathrm{GeV}$, as shown in Figure 8, whereas the signal does not present a specific structure. The $\bar{t} t$ background is thus strongly reduced by the cut on $M_{T 2}$. A significant reduction is also obtained for the SUSY backgrounds where the two leptons are produced in the decays of two different squarks, and therefore the $M_{T 2}$ distribution contains information on the kinematics of the two decays. 


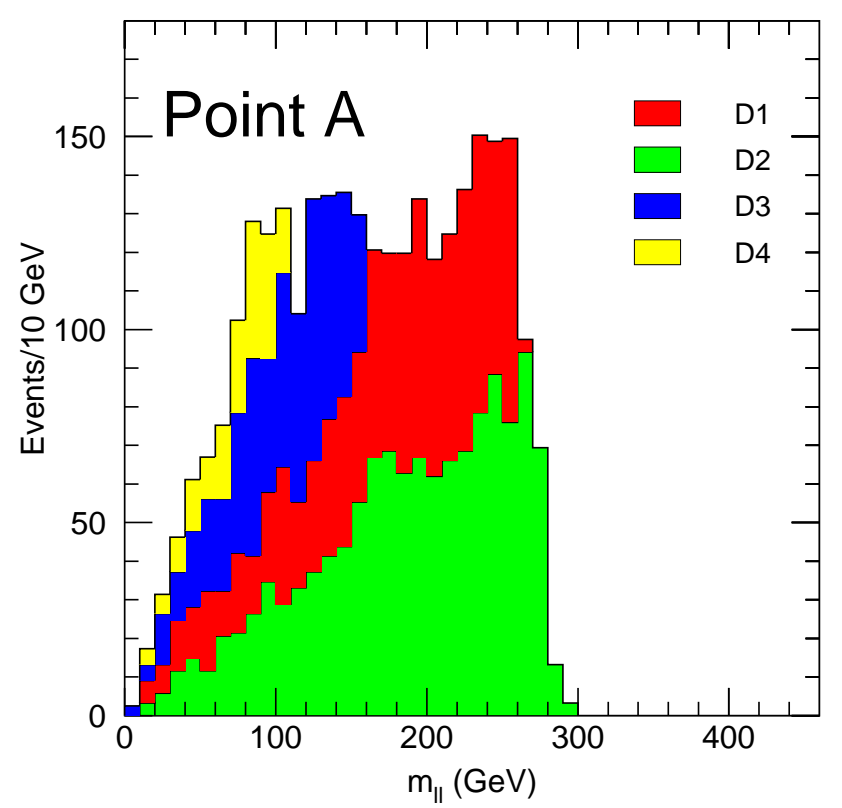

Figure 6. Point A: distribution of $m_{\ell \ell}$ before cuts for the decay chains [D1] (red), [D2] (green), [D3] (blue) and [D4] (yellow).

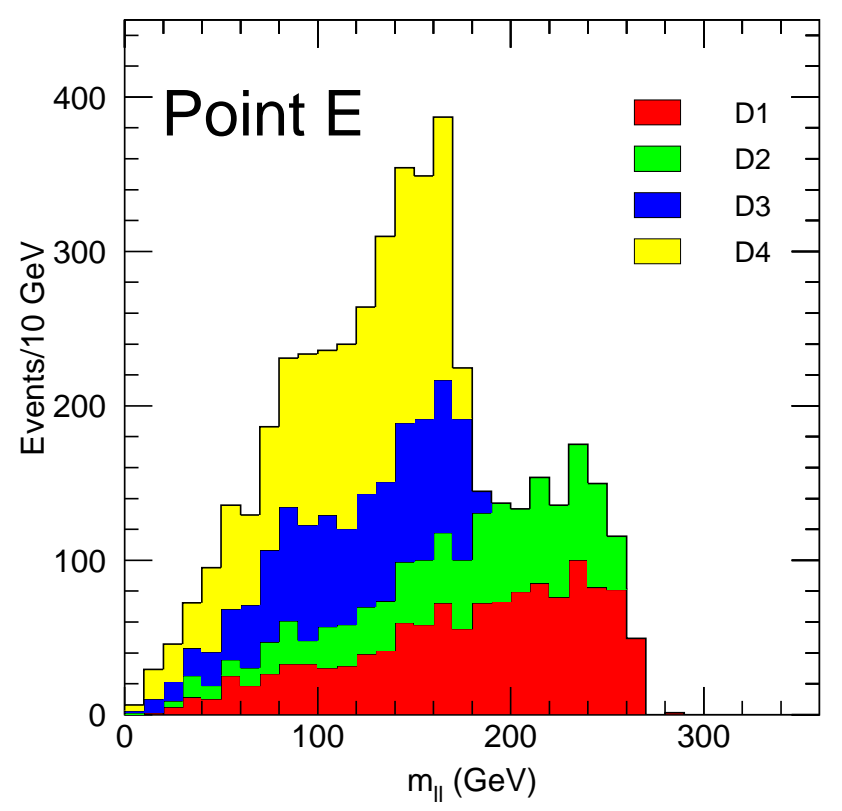

Figure 7. Point E: distribution of $m_{\ell \ell}$ before cuts for the decay chains [D1] (red), [D2] (green), [D3] (blue) and [D4] (yellow). 


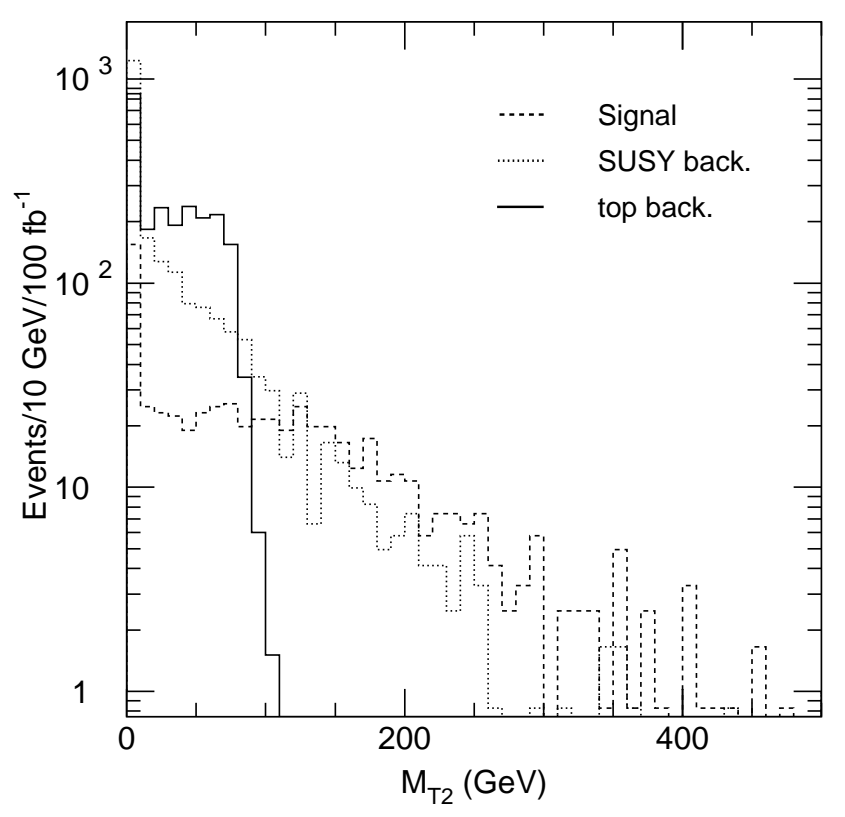

Figure 8. Point A: distribution of the $M_{T 2}$ variable respectively for: signal (dashed line), SUSY background (dotted line), $\bar{t} t$ background (full line).

After these cuts, the SM background is 70 events, of which 42 are from $\bar{t} t$ and 28 from $Z+$ jets for an integrated luminosity of $100 \mathrm{fb}^{-1}$. This is well below the SUSY background which, for the considered points is between $\sim 250$ and $\sim 600$ events. The $Z+$ jets background is however mainly made of flavour-correlated lepton pairs from off-shell $Z^{*} / \gamma^{*}$ production, and still pollutes the signal after opposite-flavour lepton subtraction. By raising the cut on $m_{\ell \ell}$ to $120 \mathrm{GeV}$, this background is reduced to less than 6 events. It is however worth to point out that for a complete estimate a study based on a full $Z+4$ jets event generator will be needed. The efficiency for the signal after cuts is between 20 and $30 \%$. The requirement of exactly two leptons allows for a cleaner subtraction of the background for uncorrelated leptons at the price of a significant loss in signal statistics.

The invariant mass distribution for OS-SF and OS-OF lepton pairs is shown in Figures 9, 10, 12 and 13 for Points $\mathrm{A}$ and $\mathrm{E}$ respectively, both in logarithmic and in linear scale. A clear excess is seen in the OS-SF spectra. The $m_{\ell \ell}$ spectra after subtraction are shown with errors including the fluctuation from the subtraction procedure, superimposed on the expected signal shape in Figures 11 and 14 for Points A and E respectively. We define here the statistical significance as $S / \sqrt{B}$, where $S$ is the number of signal events and $B$ the number of background events. In order to evaluate the significance of the signal, for each considered model point a different mass window was chosen, with the aim of maximizing the significance. For each point the number of signal and background events inside the selected mass window are listed in 


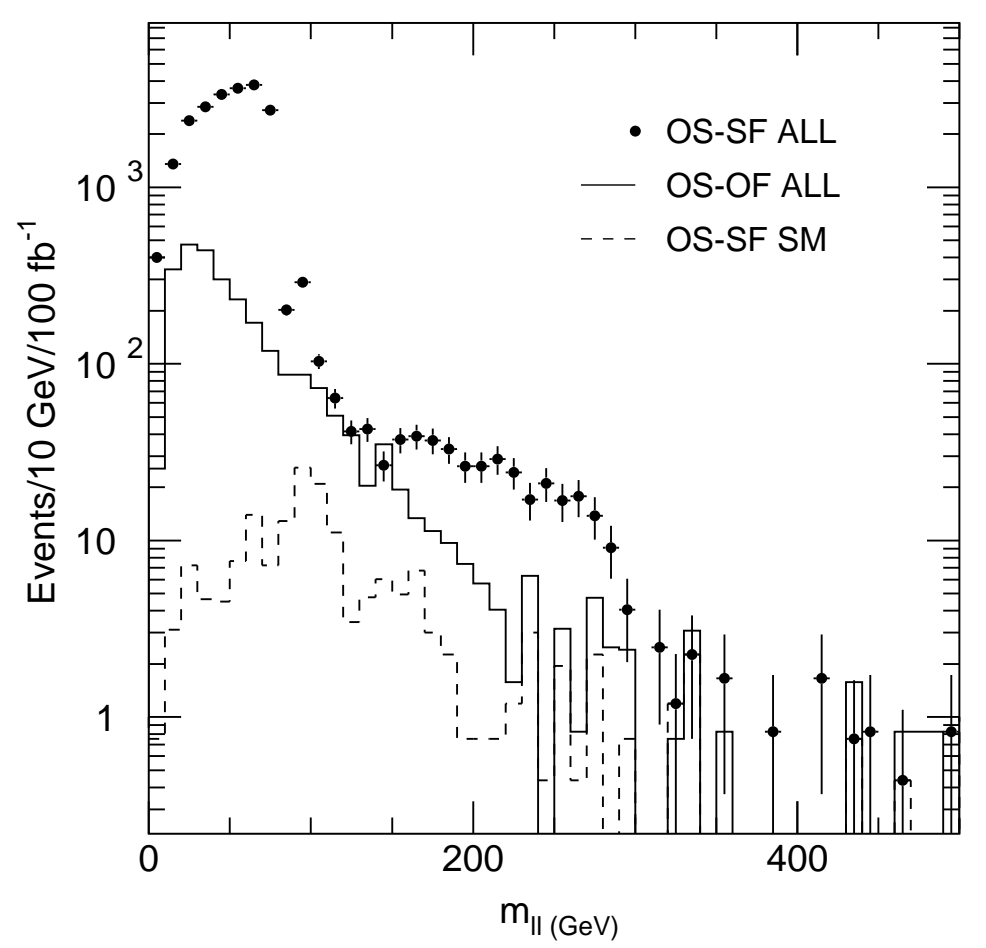

Figure 9. Point A: Invariant mass spectrum respectively for: OS-SF leptons total (full dots), OS-OF leptons total (full line), OS-SF leptons Standard Model (dashed line). All cuts are applied, except the lower cut on $m_{\ell \ell}$.

Table 2. Results after cuts for the 12 example models. We show in column 2 the number of signal events, in 3 the statistical significance, in 4 the total background, in 5 the SM background, and in 6 the invariant mass window chosen to optimize the significance. The assumed luminosity is $100 \mathrm{fb}^{-1}$.

\begin{tabular}{lrrrll}
\hline Point & \multicolumn{1}{c}{$N_{e v}$ Signal } & Significance & Total bg. & SM bg. & Window $(\mathrm{GeV})$ \\
\hline A & $259 \pm 21$ & 12.3 & 92 & 27 & $150-290$ \\
B & $242 \pm 23$ & 10.5 & 150 & 27 & $150-300$ \\
C & $125 \pm 16$ & 7.8 & 68 & 13 & $200-350$ \\
D & $89 \pm 14$ & 6.4 & 53 & 13 & $200-350$ \\
E & $420 \pm 31$ & 13.5 & 265 & 31 & $140-260$ \\
F & $79 \pm 15$ & 5.3 & 72 & 10 & $200-300$ \\
G & $33 \pm 9$ & 3.7 & 20 & 4 & $260-320$ \\
H & $228 \pm 30$ & 7.6 & 332 & 27 & $150-290$ \\
I & $188 \pm 34$ & 5.5 & 470 & 35 & $130-270$ \\
L & $71 \pm 31$ & 2.3 & 431 & 36 & $130-300$ \\
\hline
\end{tabular}

Table 2. Interpolating the significances of the studied points, an approximate region in the $\left(m_{0}, m_{1 / 2}\right)$ where the signal is detectable at the $5 \sigma$ level can be drawn, and is 


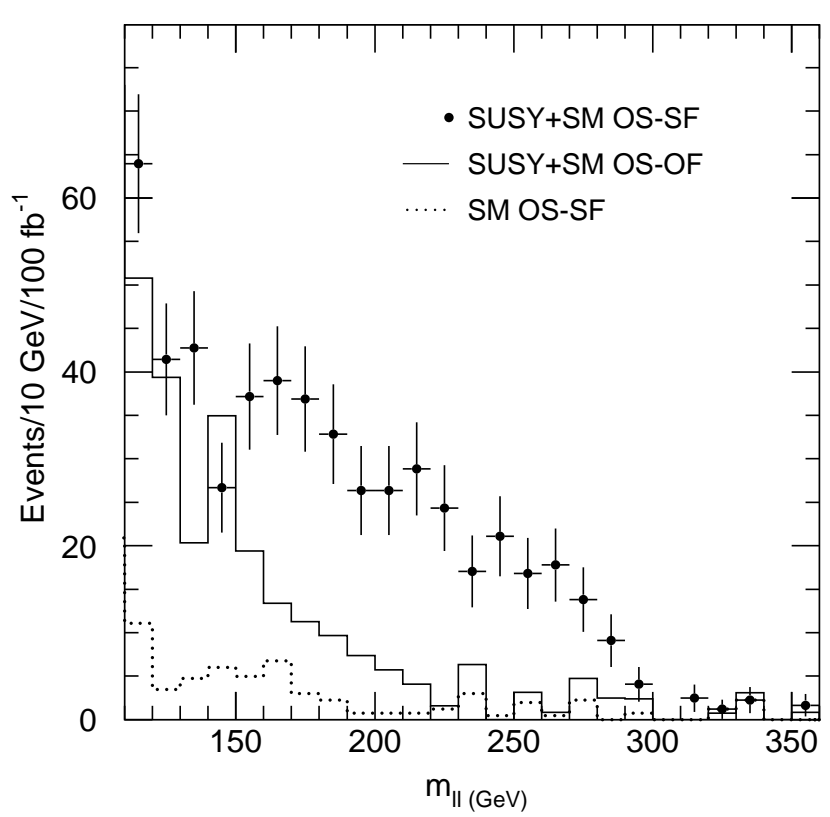

Figure 10. Point A: Invariant mass spectrum respectively for OS-SF leptons total (full dots), OS-OF leptons total (full line).

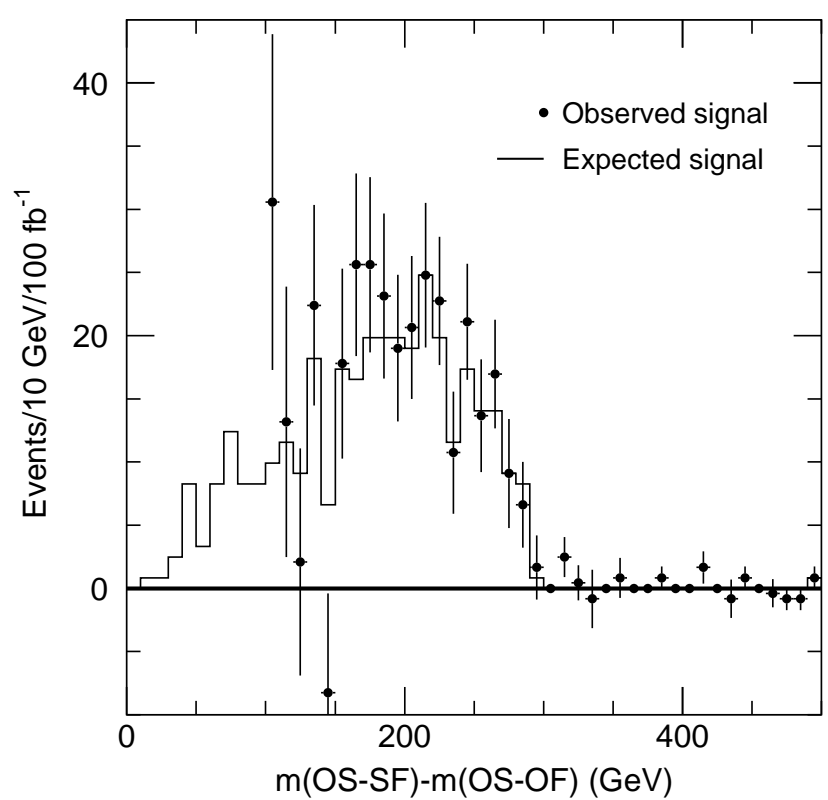

Figure 11. Point A: Invariant mass spectrum after subtracting opposite-flavour lepton pairs from same-flavour lepton pairs. The black points show the result of the subtraction, with the statistical errors for $100 \mathrm{fb}^{-1}$. The full line is the spectrum for events containing the desired decay chains. 


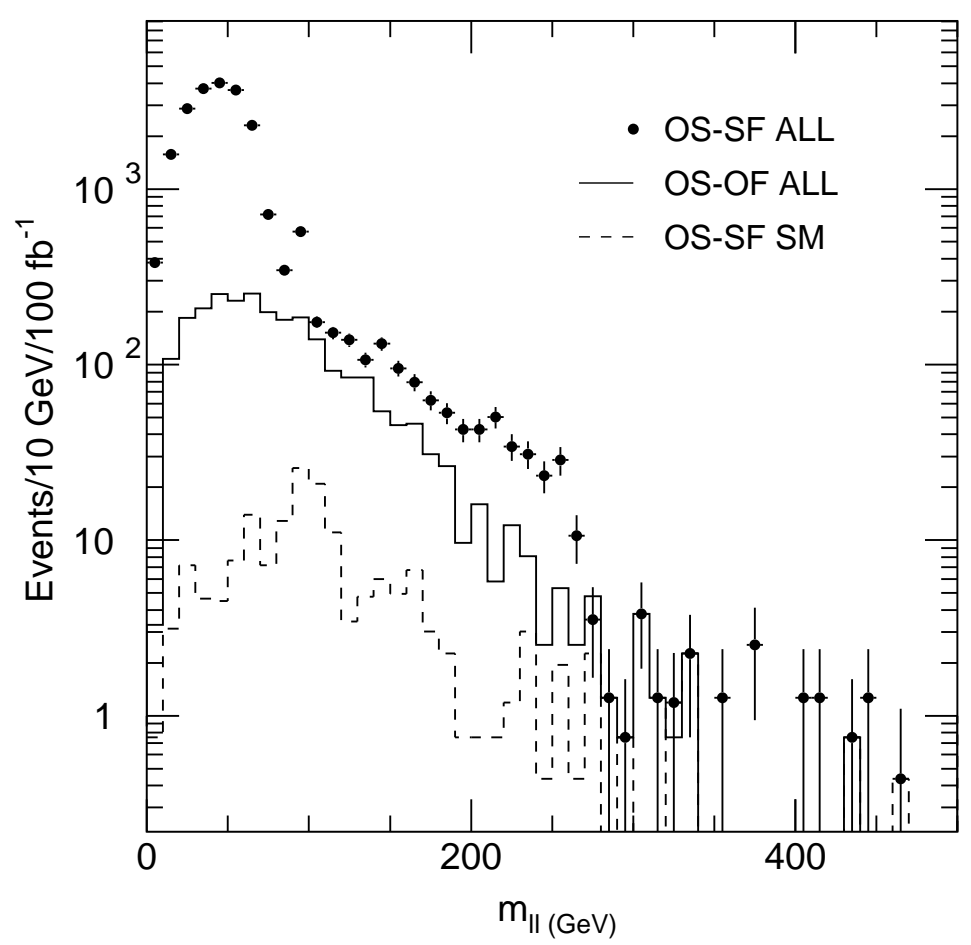

Figure 12. Point E: invariant mass spectrum respectively for for: OS-SF leptons total (full dots), OS-OF leptons total (full line), OS-SF leptons Standard Model (dashed line). All cuts are applied, except the lower cut on $m_{\ell \ell}$.

shown in Figure 15. By comparing the reach with the lines giving the regions with equal numbers of signal events, also shown in the figure, one can observe that the signal detection is much easier in the low $m_{0}$ region where $\tilde{\chi}_{4}^{0}$ dominates over the $\tilde{\chi}_{2}^{ \pm}$. This is due to the fact that the invariant mass distribution of the background falls rather quickly with increasing $m_{\ell \ell}$. Therefore the $\tilde{\chi}_{2}^{ \pm}$signal, which has edges at lower masses is much more affected by background fluctuations.

\section{Gaugino mass measurement}

A statistically significant signal from the leptonic decays of the heavier gauginos is accessible for a region of the mSUGRA parameter space, as discussed in the previous section. The excess observable in the $m_{\ell \ell}$ distribution for OS-SF pairs has an end point which is connected to the masses of the sparticles taking part in the decay through equation 2. The edge structure does, however, involve up to four independent decay channels, as discussed in Section 2, and the definition of the decay yielding the outermost edge requires additional information. For most model choices yielding a signal for the heavy gauginos, the masses of the lighter sparticles will be measured by different 


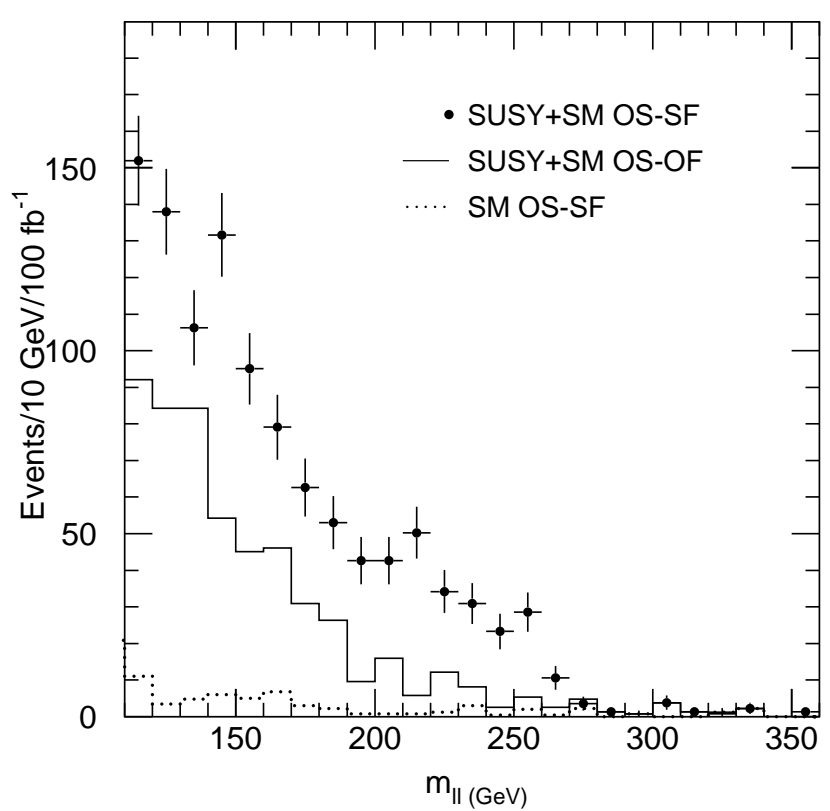

Figure 13. Point E: Invariant mass spectrum respectively for OS-SF leptons total (full dots), OS-OF leptons total (full line).

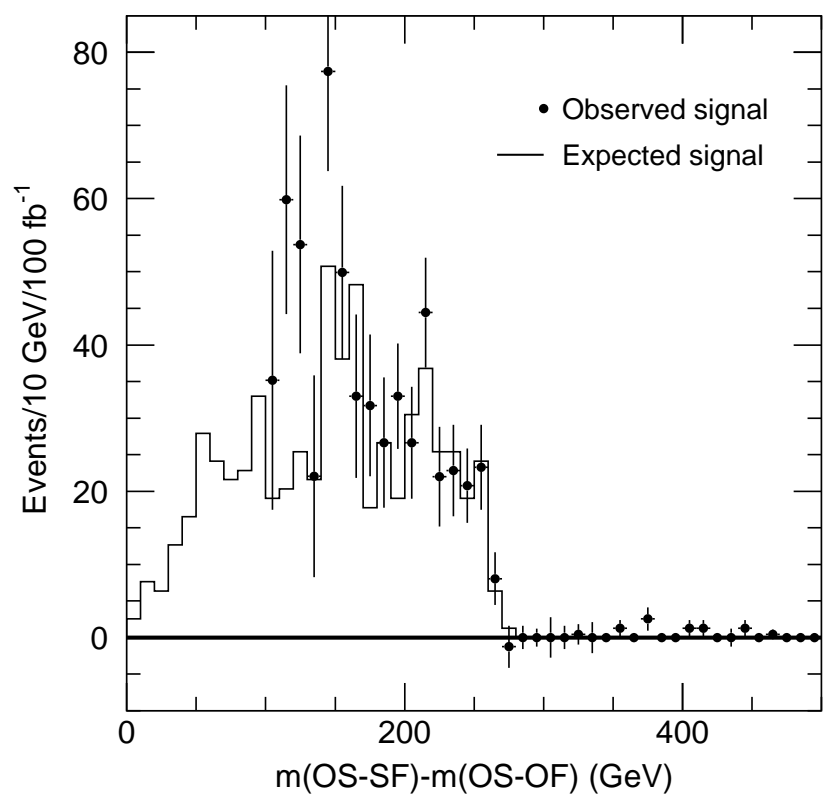

Figure 14. Point E: Invariant mass spectrum after subtracting opposite-flavour lepton pairs from same-flavour lepton pairs. The black points show the result of the subtraction, with the statistical errors for $100 \mathrm{fb}^{-1}$. The full line is the spectrum for events containing the desired decay chains. 


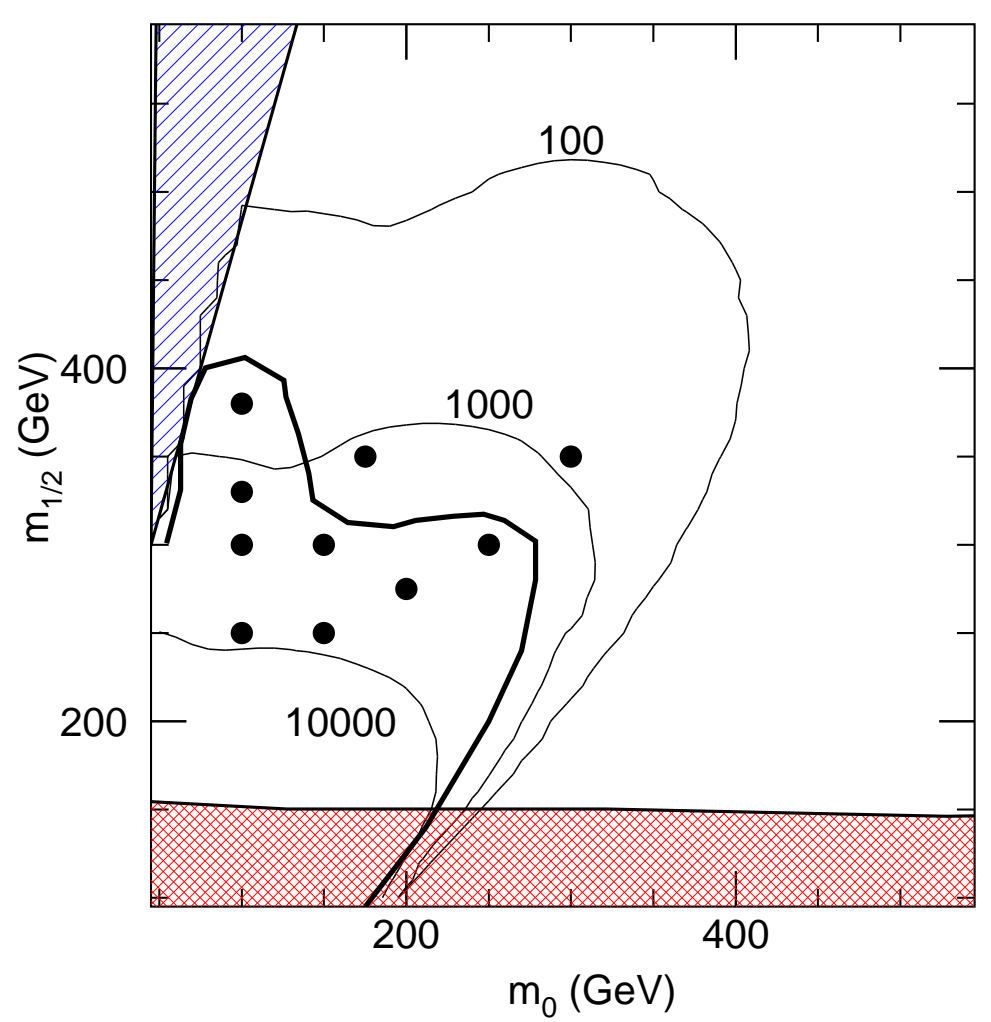

Figure 15. The region for which the $\tilde{\chi}_{4}^{0}, \tilde{\chi}_{2}^{ \pm}$signal can be observed at the $5 \sigma$ level in the $\left(m_{0}, m_{1 / 2}\right)$ plane is below and to the left of the thick line. The cross-hatched region on the lower part of the figure is excluded by experimental measurements, and the hatched region on the upper right by theory. The lines corresponding to respectively 100, 1000, and 10000 signal events are also shown in the plot. The other mSUGRA parameters are $A=0, \tan \beta=10$ and $\mu>0$. The assumed luminosity is $100 \mathrm{fb}^{-1}$.

analyses. In particular the study of the $\tilde{q}_{L}$ decays to $\tilde{\chi}_{2}^{0}$ will yield a measurement of the masses of $\tilde{\ell}_{R}, \tilde{\chi}_{1}^{0}, \tilde{\chi}_{2}^{0}[2,3]$ and probably $\tilde{\chi}_{1}^{ \pm}$. The study of direct slepton production will give information on the mass of $\tilde{\ell}_{L}$ [14], also yielding constraints on the sneutrino mass. Starting from this information, it should be possible to translate the position of the observed end-point into a constraint for the mass of either the $\tilde{\chi}_{4}^{0}$ or $\tilde{\chi}_{2}^{ \pm}$. In the most favorable cases a detailed fit will be performed on the observed excess, yielding an estimate of the relative production rates of the four channels, and the masses of the $\tilde{\chi}_{4}^{0}$ and $\tilde{\chi}_{2}^{ \pm}$. A further ambiguity in the interpretation of the results is the possibility, in a different SUSY breaking scenario than the one considered in this paper, that the detected signal is from the decay of the $\tilde{\chi}_{3}^{0}$. All these possibilities should be considered when using the results of this analysis in a global fit of the SUSY parameters.

We perform here a simple approximate estimate of the achievable statistical precision on the position of the outermost edge of the OS-SF lepton invariant mass 
spectrum. The method is the following:

- the background-subtracted signal is binned in fine mass bins (2 GeV);

- the bins are grouped in a wider sliding window $(20 \mathrm{GeV})$, for increasing values of $m_{\ell \ell} ;$

- when the content of the sliding window falls below a given number of events (5-10), the lower edge of the window is taken as the estimate of the edge position.

The choice of the binning, of the size of the window, and of the threshold values on the number of observed events of course do affect the position of the measured edge. The values given above are approximately appropriate for Point $\mathrm{A}$ and for the considered luminosity $\left(100 \mathrm{fb}^{-1}\right)$. A detailed understanding of the lepton momentum resolution and a careful simulation of the expected edge shape are needed to translate the measured numbers into an actual edge measurement.

The statistical precision of the method can be studied through a set of Monte Carlo experiments. For each experiment the signal and background invariant mass distributions are obtained by fluctuating the distributions obtained from the detailed event simulation according to Poisson statistics. The end point of the distribution is then evaluated by applying the algorithm described above to each Monte Carlo experiment.

This method was applied to Points A and E. We show in Figure 16 for Point A the distribution of the measured edge value for a set of 10k experiments respectively for thresholds of 5 (left) and 10 (right) events. In a small number of cases, for extreme high fluctuations of the background, the cut at 5 events yields an evaluation well above the nominal value. Exploiting the comparison with the estimate for a cut of 10 events, the fluctuations can be detected as such, and the statistical error on the $\tilde{\chi}_{4}^{0}$ edge can be evaluated to be $\sim 4 \mathrm{GeV}$ for an integrated luminosity of $100 \mathrm{fb}^{-1}$. Comparable results are obtained for Point E.

\section{Conclusions}

An excess in the invariant mass of (OSSF-OSOF) lepton pairs due to squark decay into $\tilde{\chi}_{4}^{0}$ and $\tilde{\chi}_{2}^{ \pm}$can be observed over a significant part of the mSUGRA parameter space for 1 year at high luminosity at the LHC.

After cuts the signal is at most a few hundred events, and its detectability relies on the possibility of subtracting the backgrounds exploiting the correlation of the lepton flavours in the signal.

The end-point of the lepton-lepton invariant mass distribution for the observed signal can be measured with a precision of a few GeV. Using this measurement, constraints on the masses of the heavier gauginos can be extracted. Additional information is available from the shape and the number of events in the observed excess. By including measurements from other measured decay chains, it may be possible to disentangle the complex structure of the signal to further constrain the parameters of the gaugino mixing matrix. 

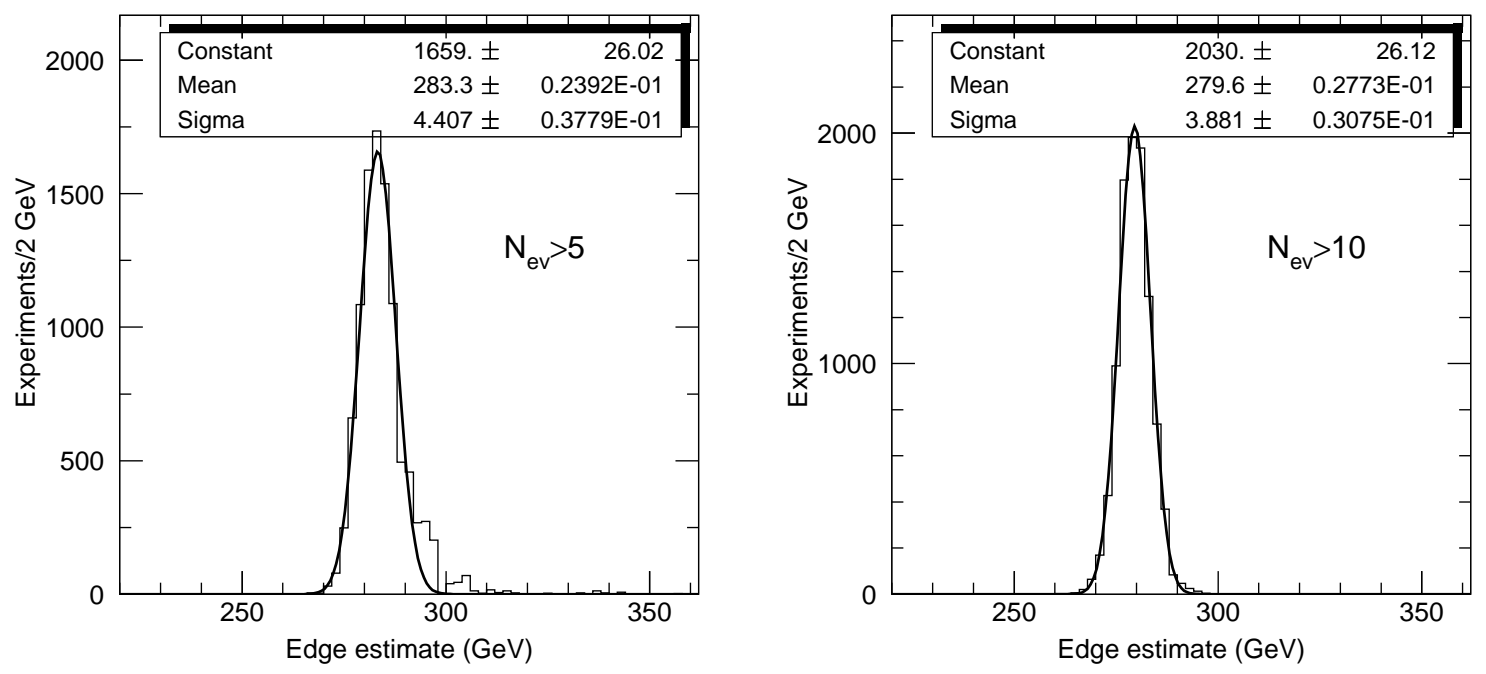

Figure 16. Distribution of estimate of edge position for Point A, requiring respectively $>5$ (left plot) and $>10$ (right plot) events in sliding window for 10k Monte Carlo experiments. The assumed integrated luminosity is $100 \mathrm{fb}^{-1}$. The nominal position of the edge is $283 \mathrm{GeV}$.

\section{Acknowledgments}

This work has been performed within the ATLAS collaboration, and I thank collaboration members for useful discussions. I have made use of the physics analysis and simulation tools which are the result of collaboration-wide efforts.

\section{References}

[1] H. E. Haber and G. L. Kane, Phys. Rept. 117 (1985) 75.

[2] The ATLAS Collaboration, 'ATLAS Detector and Physics Performance Technical Design Report', ATLAS TDR 15, CERN/LHCC/99-15 (1999).

[3] B. C. Allanach, C. G. Lester, M. A. Parker and B. R. Webber, JHEP 0009 (2000) 004 [arXiv:hep$\mathrm{ph} / 0007009]$.

[4] H. Baer, F. E. Paige, S. D. Protopopescu, X. Tata, arXiv:hep-ph/9305342; arXiv:hep-ph/980432; arXiv:hep-ph/0001086.

[5] S. Y. Choi, J. Kalinowski, G. Moortgat-Pick and P. M. Zerwas, Eur. Phys. J. C 22 (2001) 563 [Addendum-ibid. C 23 (2002) 769] [arXiv:hep-ph/0108117].

[6] W. Beenakker, R. Hopker and M. Spira, arXiv:hep-ph/9611232.

[7] G. Marchesini, B.R. Webber, G. Abbiendi, I.G. Knowles, M.H. Seymour and L. Stanco, Comput. Phys. Commun. 67 (1992) 465;

G. Corcella, I.G. Knowles, G. Marchesini, S. Moretti, K. Odagiri, P. Richardson, M.H. Seymour and B.R. Webber, JHEP 0101 (2001) 010 [arXiv:hep-ph/9912396].

[8] T. Sjöstrand, P. Edén, C. Friberg, L. Lönnblad, G. Miu, S. Mrenna, E. Norrbin, Comput.Phys.Commun. 135 (2001) 238.

[9] E. Richter-Was, D. Froidevaux, L. Poggioli, 'ATLFAST 2.0: a fast simulation package for ATLAS', ATLAS Internal Note ATL-PHYS-98-131 (1998). 
[10] M. L. Mangano, M. Moretti, F. Piccinini, R. Pittau and A. D. Polosa, JHEP 0307 (2003) 001 [arXiv:hep-ph/0206293].

[11] B. C. Allanach et al., in Proc. of the APS/DPF/DPB Summer Study on the Future of Particle Physics (Snowmass 2001) ed. N. Graf, Eur. Phys. J. C 25 (2002) 113 [eConf C010630 (2001) P125] [arXiv:hep-ph/0202233].

[12] C. G. Lester and D. J. Summers, Phys. Lett. B 463 (1999) 99 [arXiv:hep-ph/9906349].

[13] A. Barr, C. Lester and P. Stephens, J. Phys. G 29 (2003) 2343 [arXiv:hep-ph/0304226].

[14] E. Lytken, Ph.D. Thesis, Copenhagen University, 2003. 